\title{
GATA binding protein 6 (GATA6) is co-amplified with PIK3CA in patients with esophageal adenocarcinoma and is linked to neoadjuvant therapy
}

\author{
Patrick Sven Plum ${ }^{1,2,3,4} @$. Heike Löser ${ }^{2,5}$. Thomas Zander ${ }^{4,6} \cdot$ Ahlem Essakly $^{5}$. Christiane J. Bruns $s^{1,4}$. \\ Axel M. Hillmer ${ }^{5} \cdot$ Hakan Alakus $^{1,4} \cdot$ Wolfgang Schröder $^{1,4} \cdot$ Reinhard Büttner $^{5} \cdot$ Florian Gebauer $^{1,4}$. \\ Alexander Quaas ${ }^{2,4,5}$
}

Received: 22 October 2020 / Accepted: 27 November 2020 / Published online: 10 December 2020

(c) The Author(s) 2020

\begin{abstract}
Purpose Driver mutations are typically absent in esophageal adenocarcinoma (EAC). Mostly, oncogenes are amplified as driving molecular events (including GATA6-amplification in 14\% of cases). However, only little is known about its biological function and clinical relevance.

Methods We examined a large number of EAC $(n=496)$ for their GATA6 amplification by fluorescence in situ hybridization (FISH) analyzing both primary resected $(n=219)$ and neoadjuvant treated EAC $(n=277)$. Results were correlated to clinicopathological data and known mutations/amplifications in our EAC-cohort.

Results GATA6 amplification was detectable in 49 (9.9\%) EACs of our cohort. We observed an enrichment of GATA6positive tumors among patients after neoadjuvant treatment $(12,3 \%$ amplified tumors versus $6,8 \%$ in the primary resected group; $p=0.044)$. Additionally, there was a simultaneous amplification of PIK3CA and GATA6 $(p<0.001)$ not detectable when analyzing other genes such as $E G F R, E R B B 2, K R A S$ or $M D M 2$. Although we did not identify a survival difference depending on GATA6 in the entire cohort $(p=0.212)$, GATA6 amplification was associated with prolonged overall survival among patients with primary surgery (median overall-survival 121.1 vs. 41.4 months, $p=0.032$ ). Multivariate cox-regression analysis did not confirm GATA6 as an independent prognostic marker, neither in the entire cohort $(p=0.210)$, nor in the subgroup with ( $p=0.655)$ or without pretreatment $(p=0.961)$.

Conclusions Our study investigates the relevance of GATA6 amplification on a large tumor collective, which includes primary resected tumors and the clinically relevant group of neoadjuvant treated EACs. Especially in the pretreated group, we found an accumulation of GATA6-amplified tumors (12.3\%) and a frequent co-amplification of PIK3CA. Our data suggest an increased resistance to radio-chemotherapy in GATA6-amplified tumors.
\end{abstract}

Keywords GATA6 · PIK3CA · Esophageal adenocarcinoma · EAC $\cdot$ Prognosis $\cdot$ Biomarker $\cdot$ Neoadjuvant therapy . Treatment response $\cdot$ Neoadjuvant treatment

Patrick Sven Plum

Patrick.plum@uk-koeln.de

1 Department of General, Visceral, Cancer, and Transplantation Surgery, University of Cologne, Faculty of Medicine, and University Hospital Cologne, Kerpener Straße 62, 50937 Cologne, Germany

2 Gastrointestinal Cancer Group Cologne (GCGC), Cologne, Germany

3 Else Kröner Forschungskolleg Cologne "Clonal Evolution in Cancer", Cologne, Germany
4 Centre for Integrated Oncology (CIO), Cologne Bonn, Cologne, Germany

5 Institute of Pathology, University of Cologne, Faculty of Medicine, and University Hospital Cologne, Kerpener Straße 62, 50937 Cologne, Germany

6 Department of Internal Medicine I, University of Cologne, Faculty of Medicine, and University Hospital Cologne, Kerpener Straße 62, 50937 Cologne, Germany 


\section{Introduction}

Even today, esophageal adenocarcinoma (EAC) is a devastating gastrointestinal malignancy with an overall fiveyears survival ranging from 15 to $20 \%$ (DeSantis et al. 2014; Rustgi and El-Serag 2014; Coleman et al. 2018) and still increasing incidences (Arnold et al. 2017). In the recent past, efforts focused on developing more effective multimodal treatment concepts including neoadjuvant chemoradiation or perioperative chemotherapy (Al-Batran et al. 2008; van Hagen et al. 2012). Therapeutic decisions are based on mere clinical parameters deriving from staging examinations and success of neoadjuvant therapy is evaluated depending on the degree of therapeutic response towards this treatment (Shapiro et al. 2015; Al-Batran et al. 2019). However, not all patients benefit from this still very standardized treatment routines, developing only significant toxic side effects. This is the case in $35 \%$ of patients undergoing chemoradiation and 39\% of patients under chemotherapy (Ronellenfitsch et al. 2016; den Bakker et al. 2017). This clinical dilemma is due to the fact that EAC is a genetic extremely heterogenous disease. Its mutational burden is enormous (Mourikis et al. 2019; von Loga et al. 2020) and EAC is often associated with a high chromosomal instability (Frankell et al. 2019). Whole exome sequencing revealed TP53, CDKN2A, SMAD4, ARIDIA, VEGFA, CCNE1 and PIK3CA to be among those genes most frequently affected (Dulak et al. 2013; Cancer Genome Atlas Research Network et al. 2017). Another common phenomenon is the principle of genetical amplifications [also known as copy number alterations (CNAs)]. According to recent data analyzing the genetic landscape of 551 EACs these amplifications mostly occur in KRAS (19\%), c-MYC (19\%), HER2 (18\%), CCND1 (14\%) and GATA6 (14\%) (Frankell et al. 2019). However, only little is known about the function of GATA6 amplification within this entity.

GATA binding protein 6 (GATA6) belongs to the GATA family comprising of the members GATA1-6 and its gene is located on chromosome 18 (q11.1 q11.2) within the human genome (Suzuki et al. 1996). During embryogenesis, GATA6 is highly expressed within the endoderm and mesoderm (Carrasco et al. 2012) as it is essential for the development of different tissues such as adrenal gland and the central nervous system (Jimenez et al. 2003; Kamnasaran and Guha 2005). Being a transcriptional factor, dysregulation of GATA6 can also result in pathological changes and it was demonstrated that GATA6 alterations implicated in several malignancies such as non-small lung cancer (NSCLC), gastric cancer, cholangiocarcinoma, pancreatic adenocarcinoma or colorectal adenocarcinoma (Zhong et al. 2011; Shen et al. 2013; Tian et al. 2013; Van
Baal et al. 2013; Ma et al. 2019). For esophageal adenocarcinoma, it has been shown in a study including 85 EACs that gene amplification of GATA6 affected the patients' survival in a negative manner (Lin et al. 2012). During the development of Barrett's esophagus and the following malignant transformation, the expression of GATA6 is successively increasing resembling its impact on the progression of the disease (Pavlov et al. 2015).

Aim of the current study was to analyze the relevance and frequency of GATA6 amplification in a large cohort of EAC patients and the consecutively correlation with clinical, pathological and molecular parameters as well as the patients' survival.

\section{Materials and methods}

\section{Patients and tumor samples}

Analysis was performed on 496 patients with esophageal adenocarcinoma who either underwent primary surgical resection or resection after neoadjuvant treatment between 1999 and 2017 at the Department of General, Visceral, Cancer and Transplant Surgery, University of Cologne, Germany. All patients underwent primary staging including contrast-enhanced computed tomography, esophagoduodenoscopy, endoscopic ultrasound and physical examination. Patients who qualified for multimodal treatment because of locally advanced tumors ( $\mathrm{cT}>2)$ or suspected locoregional lymph node metastases $(\mathrm{cN}+)$ received neoadjuvant chemoradiation (van Hagen et al. 2012) or chemotherapy (Donohoe and Reynolds 2017). The standardized surgical procedure was transthoracic en-bloc esophagectomy with two-field lymphadenectomy of the abdominal and mediastinal lymph nodes, reconstruction via gastric pull-up and intrathoracic anastomosis (Ivor-Lewis esophagectomy). The abdominal part was predominantly performed via laparoscopy while thoracotomy was open surgery (hybrid esophagectomy). For more technical details we refer to previous publications (Plum et al. 2018) and other authors (Mariette et al. 2019). Informed consent and ethical approval were obtained from all participating patients. This retrospective study was performed according to the criteria of the ethics committee of the University Hospital of Cologne (No. 13-091 and 10-242) and in accordance with the relevant version of the Helsinki Declaration. Clinical data was collected prospectively within the department according to a standardized protocol. During the first two years, clinical follow-up of patients was performed in the out-patient clinics every three months, followed by annual exams. These included clinical evaluation, abdominal ultrasound, chest X-ray and additional diagnostic procedures as required. 
Single-spot tissue microarrays (TMA) were constructed from all surgical specimens for fluorescence in-situ hybridization (FISH) and immunohistochemical analysis. The exact procedure has been described before (Simon et al. 2005; Helbig et al. 2016). In principle, tissue cylinders with a diameter of $1.2 \mathrm{~mm}$ each were punched from the selected tumor tissue blocks (donor blocks) via a self-constructed semi-automated precision instrument and embedded on an empty paraffin block (recipient block). Four $\mu$ m sections of the resulting TMA blocks were transferred to an adhesive coated slide system (Instrumedics Inc., Hackensack, NJ, USA) for following FISH or immunohistochemistry. Amplification of GATA6 (via FISH) was correlated with molecular profiles of these EAC samples including assessments of ARIDA $1 A$ loss, TP53 mutations as well as ERBB2, c-MYC, KRAS and PIK3CA amplifications.

\section{Fluorescence in-situ hybridization (FISH) of GATA6}

Fluorescence in-situ hybridization (FISH) analysis for the evaluation of GATA6 gene copy numbers was performed with GATA6-20-GR Probe (Empire Genomics, New York, NY, USA) and the Zytolight centromere 18 (CEN18) Probe (Zytovision Bremerhaven, Germany) on the resulting TMA slides. For PIK3CA gene amplification analysis, the Zytolight SPEC PIK3CA/CEN3 Dual Probe Kit (Zytovision, Germany) was used according to the manufacturers' protocol. Three $\mu \mathrm{m}$ tissue sections on slides (SuperFrost Plus) were mounted by heating, followed by deparaffinization, protease digestion, washing steps (VP2000 processor system, Abbott Molecular, Wiesbaden, Germany) and hybridization at $37{ }^{\circ} \mathrm{C}$ overnight with the FISH Probe. The slides were stained with DAPI before analysis. Cases were further evaluated only when normal tissue nuclei displayed one or two clearly distinct signals of green GATA6 and orange CEN18. Tumor tissue was scanned for amplification hot spots of GATA6 signals using $\times 63$ objective (DM5500 fluorescent microscope; Leica). This reading strategy followed that of the c-MYC-FISH probe to evaluate areas of cluster amplification. GATA6 amplification was defined as gene copy cluster $>50 \%$ of the tumor cells, respectively, gene copy number $>6$ per cell. For PIK3CA reading strategy followed the recommendations of previous studies amplification such as (Essakly et al. 2020).

\section{Immunohistochemistry}

Immunohistochemistry (IHC) was performed on TMA slides using the following antibodies against MHC1, PDL1, LAG3, IDO, INI, VISTA, TP53, TIM3, TUBB3, HER2, Ki67, ARIDA 1A, BRG1, BRM, Met1 and c-MYC as already published by our group (Becker et al. 2015; Loeser et al.
2019; Plum et al. 2019; Essakly et al. 2020; Gebauer et al. 2020; Wagener-Ryczek et al. 2020; Schiffmann et al. 2020).

\section{Statistical analysis}

SPSS Statistics for Mac (Version 21, SPSS) was used for statistical analysis. Interdependence between stainings and clinical data were calculated using the chi-squared and Fisher's exact tests, and displayed by cross-tables. Survival curves were plotted using the Kaplan-Meier method and analyzed using the log-rank test. All tests were two-sided. $p$ values $<0.05$ were considered statistically significant.

\section{Results}

\section{Patients' baseline characteristics}

A total of 496 patients of 685 on the TMA with EAC were interpretable on the single-spot for GATA6. Reasons for non-informative cases (189 spots; $27.6 \%$ ) included lack of tissue samples or absence of unequivocal cancer tissue in the TMA spot. Clinico-pathological data were summarized within Table 1 . The majority of patients were male (male: $n=437 ; 88.1 \%$ versus female: $n=59 ; 11.9 \%)$. The median age was 65.2 years (range 33.6-85.6 years) at the time point of diagnosis. More than half of the patient cohort $(n=277$; $55.8 \%$ ) underwent multimodal treatment (including either chemoradiation or chemotherapy before surgical resection) while $219(44.2 \%)$ patients received primary surgery.

\section{GATA6 amplification in esophageal adenocarcinoma and correlation to clinico-pathological data}

Considering the entire patient cohort, GATA6 amplification was detectable via FISH in 49 patients (9.9\%) within an intranuclear pattern (compare Fig. 1). There was no significant correlation between such clinico-pathological parameters such as sex, age, grading, (y)pT-category, (y) pN-category or UICC-stage (see Table 1). However, GATA6 amplification was correlated with the status of neoadjuvant treatment ( $p=0.044)$. Patients who had multimodal therapy showed in $12.3 \%$ an amplification in the FISH examination compared to $6.8 \%$ among those patients who had primary esophagectomy.

\section{GATA6 and PIK3CA co-amplification}

FISH-data of GATA6 amplification was additionally correlated with other important biomarkers in EAC like other amplified oncogenes, immune checkpoint markers such as PD-L1, LAG3, IDO, INI, VISTA or the antigen-presenting protein $\mathrm{MHC1}$, as well as additional proteins like the 
Table 1 Clinico-pathological parameters for the patient cohort

\begin{tabular}{|c|c|c|c|c|c|c|c|}
\hline \multirow[t]{2}{*}{ Factor } & \multirow[t]{2}{*}{ Total } & & \multicolumn{4}{|c|}{ GATA6 } & \multirow[t]{2}{*}{$p$ value } \\
\hline & & & \multicolumn{2}{|c|}{ Negative } & \multicolumn{2}{|c|}{ Positive } & \\
\hline \multicolumn{8}{|l|}{ Sex } \\
\hline Female & 59 & $11.9 \%$ & 53 & $89.8 \%$ & 6 & $10.2 \%$ & \\
\hline Male & 437 & $88.1 \%$ & 394 & $90.2 \%$ & 43 & $9.8 \%$ & 0.937 \\
\hline \multicolumn{8}{|l|}{ Agegroup } \\
\hline$<65$ yrs & 245 & $52,5 \%$ & 216 & $88.2 \%$ & 29 & $11.8 \%$ & \\
\hline$>65 \mathrm{yrs}$ & 222 & $47.5 \%$ & 202 & $91.0 \%$ & 20 & $9.0 \%$ & 0.319 \\
\hline \multicolumn{8}{|c|}{ Tumor stage } \\
\hline $\mathrm{pT} 1 / 2$ & 125 & $25.4 \%$ & 112 & $89.6 \%$ & 13 & $10.4 \%$ & \\
\hline $\mathrm{pT} 3 / 4$ & 368 & $74.6 \%$ & 332 & $90.2 \%$ & 36 & $9.8 \%$ & 0.842 \\
\hline \multicolumn{8}{|c|}{ Lymph node metastasis } \\
\hline pNO & 198 & $40.1 \%$ & 178 & $89.9 \%$ & 20 & $4.0 \%$ & \\
\hline $\mathrm{pN}+$ & 296 & $59.9 \%$ & 267 & $90.2 \%$ & 29 & $9.8 \%$ & 0.912 \\
\hline \multicolumn{8}{|l|}{ Grading } \\
\hline G1 & 5 & $1.4 \%$ & 5 & $1.4 \%$ & 0 & $0 \%$ & \\
\hline $\mathrm{G} 2$ & 197 & $55.5 \%$ & 178 & $90.4 \%$ & 19 & $9.6 \%$ & \\
\hline G3 & 151 & $42.5 \%$ & 137 & $90.7 \%$ & 14 & $9.3 \%$ & \\
\hline G4 & 2 & $0.6 \%$ & 2 & $0.6 \%$ & 0 & $0 \%$ & 0.746 \\
\hline \multicolumn{8}{|l|}{ UICC } \\
\hline I & 108 & $22.0 \%$ & 97 & $89.8 \%$ & 11 & $10.2 \%$ & \\
\hline II & 106 & $21.5 \%$ & 96 & $90.6 \%$ & 10 & $9.4 \%$ & \\
\hline III & 208 & $42.3 \%$ & 184 & $88.5 \%$ & 24 & $11.5 \%$ & \\
\hline IV & 70 & $14.2 \%$ & 66 & $94.3 \%$ & 4 & $5.7 \%$ & 0.567 \\
\hline \multicolumn{8}{|c|}{ Neoadjuvant therapy } \\
\hline No & 219 & $44.2 \%$ & 204 & $93.2 \%$ & 15 & $6.8 \%$ & \\
\hline Yes & 277 & $55.8 \%$ & 243 & $87.7 \%$ & 34 & $12.3 \%$ & 0.044 \\
\hline
\end{tabular}

chromatin-remodeler and SWI/SNF components ARIDA $1 A$, $B R G 1, B R M$ and oncogene amplifications like $M E T, c-M Y C$, $K R A S, E R B B 2, M D M 2$ and PIK3CA. We observed no correlation between GATA6 and most of these other biomarkers within the cohort performing the cross-table analysis (see Table 2). However, we identified co-amplification of GATA6 together with PIK3CA in $9(1.8 \%)$ patients of the entire cohort $(p<0.001)$ divided into $2(0.3 \%)$ patients of the pretreated subgroup $(p<0.001)$ and $7(1.4 \%)$ patients with primary surgery $(p=0.174)$. PIK3CA amplifications were seen in 24 patients (4.8\%) (Essakly et al. 2020). Similar amplification rates were seen within the primary surgery group $(n=11 ; 5.0 \%)$ and surgery after neoadjuvant treatment $(n=13 ; 4.7 \%)$. All details are illustrated in Table 3.

\section{GATA6 amplification is associated with a prolonged survival among patients who did not receive neoadjuvant treatment}

Considering the entire patient cohort of the present study, a significant difference between patients with and without GATA6 amplification could not be observed (median survival without GATA6 amplification: 26.1 months (95\% CI
20.4-31.7 months) versus median survival with GATA6 amplification: 37.2 months (95\% CI 29.3-45.1 months, $p=0.212$ ) (Fig. 2a). The same was true for patients receiving neoadjuvant treatment. In this subgroup, postsurgical survival was comparable between patients with and those without GATA6 amplification (median survival without GATA6 amplification: 22.3 months (95\% CI 18.2-26.4 months) versus median survival with GATA6 amplification: 31.9 months (95\% CI 28.2-35.6 months, $p=0.699$ ) (Fig. 2b). However, in patients without neoadjuvant therapy, intratumoral GATA6 amplification was associated with a prolonged overall survival (OS) compared to those tumors without this amplification (Fig. 2c) $(p=0.032)$. The median OS was 121.1 months $(95 \%$ CI not calculable) in patients with GATA6-amplified tumors in contrast to a median OS of 41.4 months $(95 \%$ CI 23.4-59.4 months, $p=0.032$ ) in patients with normal GATA6 expression.

Multivariate cox-regression analysis did not confirm GATA6 as an independent prognostic marker, neither in the entire cohort $(p=0.210)$, nor in the subgroup with ( $p=0.655)$ or without neoadjuvant treatment $(p=0.961)$ (compare Table 4 for more details). 
Fig. 1 Representative images of immunohistochemistry (IHC) and fluorescence in-situ hybridization (FISH) analysis for the evaluation of GATA6 gene copy numbers using the GATA620-GR (green) and the Zytolight centromere 18 (CEN18) (red) Probe illustrating (upper row) GATA6-positive versus (lower row) GATA6-negative esophageal adenocarcinoma. GATA6 amplification was defined as gene copy cluster $>50 \%$ of the tumor cells, respectively, gene copy number $>6$ per cell

Table 2 Correlation between GATA6 and other molecular markers within the patient cohort

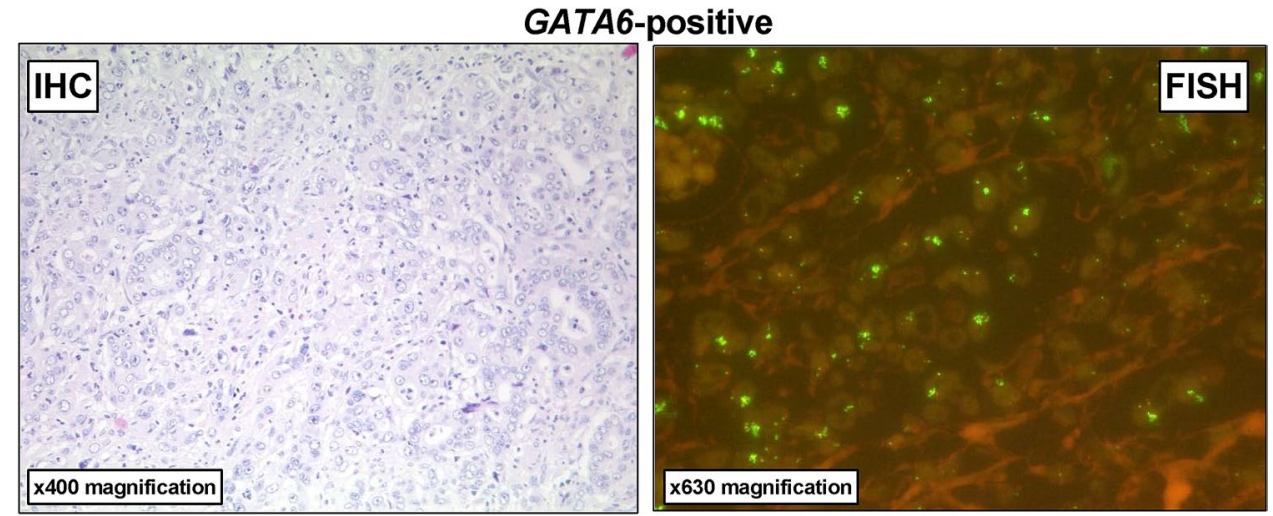

\section{GATA6-negative}

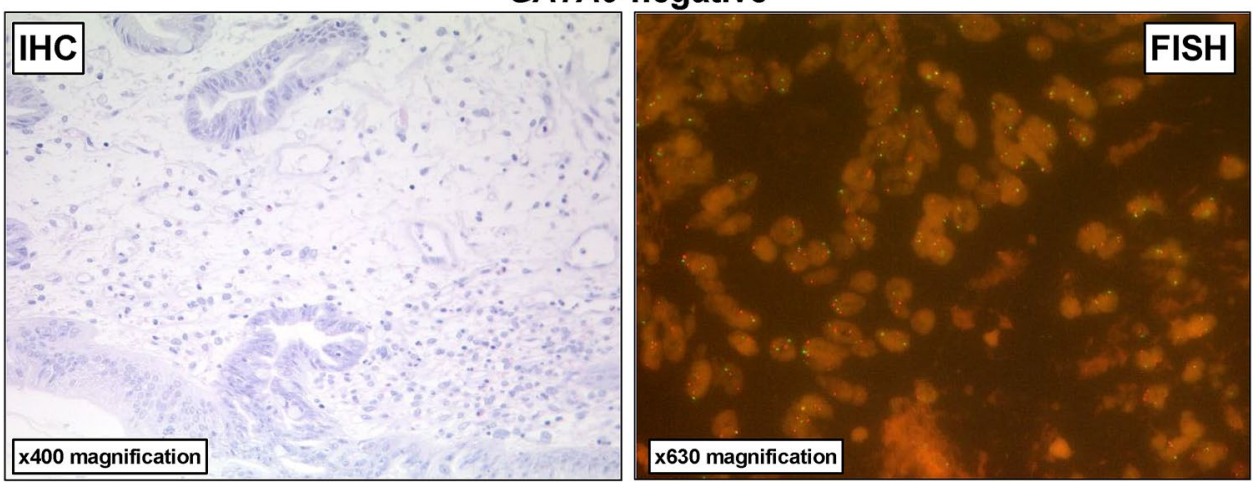

\begin{tabular}{|c|c|c|c|c|c|c|c|}
\hline \multirow[t]{2}{*}{ Factor } & \multirow[t]{2}{*}{ Total } & & \multicolumn{4}{|c|}{ GATA6 } & \multirow[t]{2}{*}{$p$ value } \\
\hline & & & \multicolumn{2}{|c|}{ Negative } & \multicolumn{2}{|c|}{ Positive } & \\
\hline \multicolumn{8}{|l|}{ HER2 } \\
\hline Normal & 300 & $87.7 \%$ & 268 & $89.3 \%$ & 32 & $10.7 \%$ & \\
\hline Mutated & 42 & $12.3 \%$ & 41 & $97.6 \%$ & 1 & $2.4 \%$ & 0.089 \\
\hline \multicolumn{8}{|l|}{ MHC1 } \\
\hline Loss & 106 & $29.4 \%$ & 101 & $95.3 \%$ & 5 & $4.7 \%$ & \\
\hline Normal & 254 & $70.6 \%$ & 227 & $89.4 \%$ & 27 & $10.6 \%$ & 0.072 \\
\hline \multicolumn{8}{|l|}{ ARIDA 1A } \\
\hline Loss & 45 & $9.5 \%$ & 44 & $97.8 \%$ & 1 & $2.2 \%$ & \\
\hline Normal & 427 & $90.5 \%$ & 381 & $89.2 \%$ & 46 & $10.8 \%$ & 0.068 \\
\hline \multicolumn{8}{|l|}{ C-myc } \\
\hline Normal & 418 & $87.8 \%$ & 379 & $90.7 \%$ & 39 & $9.3 \%$ & \\
\hline Amplified & 58 & $12.2 \%$ & 48 & $82.8 \%$ & 10 & $17.2 \%$ & 0.063 \\
\hline \multicolumn{8}{|l|}{ KRAS } \\
\hline Normal & 402 & $82.4 \%$ & 366 & $91.0 \%$ & 36 & $9.0 \%$ & \\
\hline Mutated & 86 & $17.6 \%$ & 73 & $84.9 \%$ & 13 & $15.1 \%$ & 0.084 \\
\hline \multicolumn{8}{|l|}{ PIK3CA } \\
\hline Normal & 415 & $94.5 \%$ & 379 & $91.3 \%$ & 36 & $8.7 \%$ & \\
\hline Mutated & 24 & $5.5 \%$ & 15 & $62.5 \%$ & 9 & $37.5 \%$ & $<0.001$ \\
\hline
\end{tabular}


Table 3 Correlation between GATA6 and PIK3CA within the patient cohort

\begin{tabular}{|c|c|c|c|c|c|c|c|c|}
\hline \multirow[t]{2}{*}{ Factor } & & \multirow[t]{2}{*}{ Total } & & \multicolumn{4}{|c|}{ GATA6 } & \multirow[t]{2}{*}{$p$ value } \\
\hline & & & & \multicolumn{2}{|c|}{ Negative } & \multicolumn{2}{|c|}{ Positive } & \\
\hline \multicolumn{9}{|c|}{ Entire cohort } \\
\hline \multirow[t]{2}{*}{ PIK3CA } & Normal & 415 & $94.5 \%$ & 379 & $91.3 \%$ & 36 & $8.7 \%$ & \\
\hline & Amplified & 24 & $5.5 \%$ & 15 & $62.5 \%$ & 9 & $37.5 \%$ & $<0.001$ \\
\hline \multicolumn{9}{|c|}{ Patients without neoadjuvant treatment } \\
\hline \multirow[t]{2}{*}{ PIK3CA } & Normal & 186 & $94.4 \%$ & 173 & $93.0 \%$ & 13 & $7.0 \%$ & \\
\hline & Amplified & 11 & $5.6 \%$ & 9 & $81.8 \%$ & 2 & $18.2 \%$ & 0.174 \\
\hline \multicolumn{9}{|c|}{ Patients with neaodjuvant treatment } \\
\hline \multirow[t]{2}{*}{ PIK3CA } & Normal & 229 & $94.6 \%$ & 206 & $90.0 \%$ & 23 & $10.0 \%$ & \\
\hline & Amplified & 13 & $5.4 \%$ & 6 & $46.2 \%$ & 7 & $53.8 \%$ & $<0.001$ \\
\hline
\end{tabular}

\section{Discussion}

In the current study, we focused on the frequency and clinical relevance of GATA6 amplification within a large EAC $(n=496)$ cohort by performing FISH-analysis. We identified gene amplification of GATA6 in up to $12,6 \%$ of patients. However, it had no correlation to clinico-pathological parameters such as sex, age, grading, pT-category, pNcategory or UICC-stage. Interestingly, there was a positive correlation between the amplification of GATA6 and multimodal treatment since patients after neoadjuvant therapy more frequently showed corresponding amplification compared to patients who primarily underwent surgical resection $(p=0.044)$. Additionally, distinct subgroup analysis revealed that an influence of GATA6 on the patients' survival was present depending on a multimodal treatment concept. GATA6 amplification had no effect on the OS in those patients who received neoadjuvant treatment while in patients without neoadjuvant procedures, GATA6-positive patients had a significantly prolonged OS. Correlated with other molecular alterations/amplifications common for EAC, we observed a co-amplification of GATA6 and PIK3CA in about $1.8 \%$ of patients. This effect was detectable in both subgroups with and without neoadjuvant treatment.

Our current results considering the frequency of amplified GATA6 is consistent with previous publications by recent large genetic studies (14\%) $(n=551)$ (Frankell et al. $2019)$ or the TCGA-database $(12 \%)(n=185)$ (compare http://cancergenome.nih.gov/) focusing on this malignancy. Both studies analyze primarily operated tumors (without chemoradiation) and conclude on gene amplification using a next-generation sequencing technique. Using the fluorescence in-situ technique (FISH; gold standard for determining gene amplification) we have the possibility of a direct and reliable visualization of gene copy alterations in tumor cells. In primarily operated tumors we can detect only half of GATA6-amplified EACs (6.8\%). In our cohort there is an accumulation of GATA6 amplified tumors in the group of neoadjuvant treated tumors, which has not been considered in all studies so far. However, the vast majority of EACs are now treated neoadjuvantly. Therefore, our results may suggest that GATA6-amplified tumors induce an increased resistance to either radiotherapy or chemotherapy.

One study described a much higher frequency of amplification in $20.5 \%$ of patients. However, only 85 tumors were included in this work and amplification was observed by performing an array-based comparative genomic hybridization on 20 EACs and further validation via SNP-array analysis and quantitative real-time PCR (qRT-PCR) within the rest of the cohort (Lin et al. 2012). Contrary to this, we performed FISH-analysis which resembles the current gold standard for detection of gene copy number alterations within the daily pathological routine diagnostics.

Although GATA6 amplification is recurrent in EAC, little is known about the molecular mechanisms this transcriptional factor regulates. GATA6 amplification increases during the progression from normal esophageal squamous epithelia to Barrett's metaplasia and finally to the invasive EAC (Pavlov et al. 2015). It was experimentally validated by Van Baal et al. that BMP4, a key protein within the development of Barrett's esophagus (BE) which induces SOX9 mRNA expression and which promotor is activated by GATA6, is negatively regulated via microRNA (miR)-145 (Van Baal et al. 2013). Overexpression of miR-145 in HET1A (an esophageal squamous cell line) and BAR-T cells (a non-neoplastic Barrett's esophagus cell line) resulted in an inhibition of GATA6, BMP4 and SOX9 expression and in a reduced proliferation rate. This suggested that miRNA-145 might indirectly target BMP4 via GATA6 and impact the development of BE (Van Baal et al. 2013). Another in vitro study by Lin et al. demonstrated that ectopic expression of GATA6 increased anchorage-independent growth in immortalized Barrett's esophageal cells (Lin et al. 2012). Contrary to this, GATA6 deprivation induced apoptotic (TNF-associated) pathways in EAC cells (Lin et al. 2012). Own previous data could reveal a possible connection between Dickkopf-2 (DKK2) and GATA6 in EAC (Schiffmann et al. 2020). Nevertheless, it remained unclear how these molecules interact 
Fig. 2 Kaplan-Meier survival analysis (log-rank test) considering the median survival depending on the GATA6 status of the patients. No significant GATA6-depending survival differences were observed within a the entire cohort $(p=0.212)$ as well as $\mathbf{b}$ those patients after neoadjuvant treatment $(p=0.699)$ while the subgroup of GATA6-positive patients without neoadjuvant therapy $\mathbf{c}$ showed a significant better postsurgical survival $(p=0.032)$

on the molecular level. In pancreatic adenocarcinoma, GATA6 directly binds to the DKK2-promotor leading to a down-regulation of its expression and, therefore, reduces its suppressive effect on the oncogenic Wnt pathways (Zhong et al. 2011). Interestingly, a large genome-wide association study (GWAS) on EAC performed by the German Barrett's and Esophageal Adenocarcinoma Consortium (BEACON) including about 1065 EAC cases and 1019 controls identified variants of GATA6 to be strongly associated with the disease reflecting its central role within the tumor development (Becker et al. 2015).

The reasons for the higher frequency of GATA6 amplification among patients with neoadjuvant therapy in the current analysis are unsolved. It would be interesting to assess putative changes in the GATA6 amplification rate under therapeutic pressure. To identify dynamic alterations, prospective sample collection of initial treatment-naïve biopsies during the time point of staging followed by consecutive samples from surgical specimens of patients after neoadjuvant therapy would be necessary.

To our best knowledge, this is the first study describing a simultaneous amplification of GATA6 and PIK3CA in EAC. Confirming own previous studies (Schallenberg et al. 2020), no significant co-amplifications with other common CNAs in EAC occurred. We observed amplification of PIK3CA in $4.8 \%$ of the entire cohort with no differences between patients with or without neoadjuvant treatment as already published by our group (Essakly et al. 2020). However, 1.8\% of all patients showed an amplification of both GATA6 and PIK3CA. Chromotrypsis is a recognized oncogenic mechanism of development in EAC. By this route, a synergistic co-amplification of PIK3CA and GATA6 is well conceivable (Nones et al. 2014).

In the present study, we observed a positive prognostic relevance within the subgroup of patients who did not receive neoadjuvant treatment before surgery while the prognosis of the entire cohort was not affected by GATA6 amplification. After all, the prognosis of EAC patients is still impaired and according to our results upregulation of GATA6 does not affect this in any manner. On the first sight, this seems contradictory as GATA6 has been reported to decrease the patients survival in different malignancies (Zhong et al. 2011; Shen et al. 2013, 2019; Tian et al. 2013; Rao et al. 2019). But at second glance the results for EAC are controversial. Some studies with relatively small cohorts of patients ( $n=73$, respectively, $n=58$ ) reported
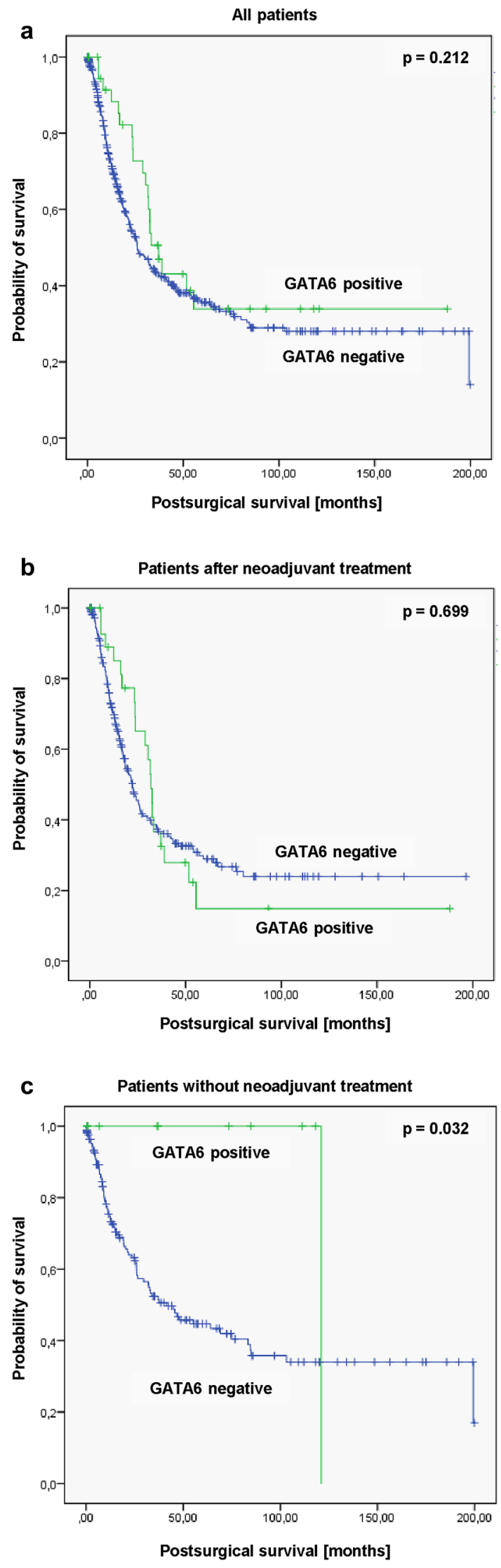


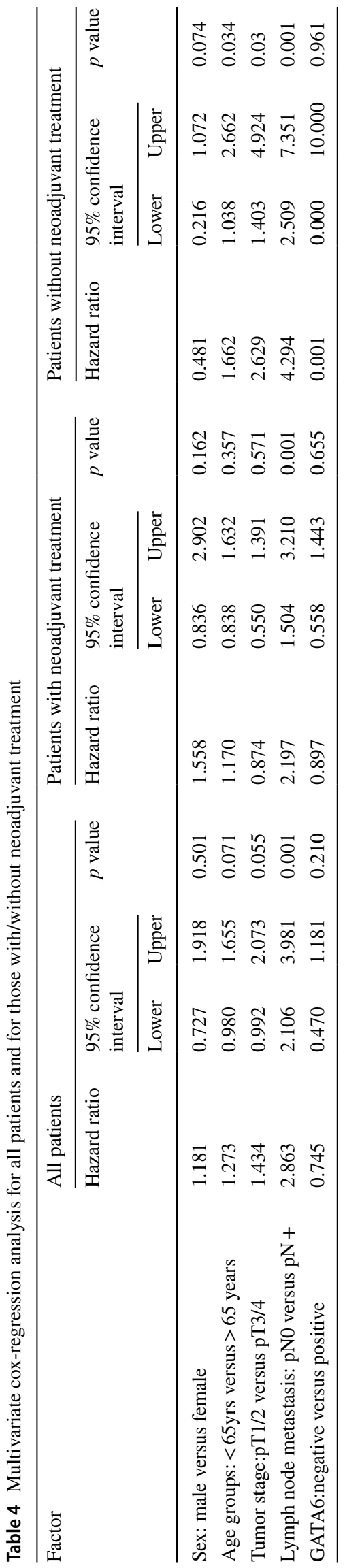

poor prognosis in patients with GATA6 amplifications (Lin et al. 2012; Toxopeus et al. 2019) while another analysis including two separated cohorts (first cohort: 130 tissue samples of normal squamous epithelium, metaplasia, dysplasia, and esophageal adenocarcinoma; second cohort: 92 esophageal adenocarcinoma) demonstrated no association between GATA6 and overall or disease-free survival in this entity (Pavlov et al. 2015). After all, our own study based on a much larger cohort size utilizing FISH as the gold standard for the detection of copy number alteration in the current pathological routine work-flow. Additionally, there are reports from gastric cancer that suggested multiple roles of GATA6 within carcinogenesis. Recently a novel suppressive function of GATA6 has been described within gastric adenocarcinoma revealing that patients with metastatic tumors had low GATA6 expression with a negative impact on the patients' survival (Liu et al. 2019). The authors illustrated that GATA6 directly targets the expression of miR-520b and that this microRNA again reduced its functional target cAMP-responsive element binding protein 1 (CREBI) leading to a suppressed cell migration, invasion and metastasis both in vitro and in vivo (Liu et al. 2019). Whether these mechanisms are also responsible for the prolonged survival within our study and why this is selectively within those patients with primary surgery remains unclear and needs further investigations.

In summary, our study identified GATA6 amplification to be significantly associated with multimodal treatment concepts in EAC and to be of prognostic impact for at least those patients with primary surgery. This might indicate an increased resistance to radio-chemotherapy in GATA6-amplified tumors. For the first time, simultaneous co-amplification of GATA6 and PIK3CA has been observed within this malignancy. Despite our large cohort, the resulting subgroups for further analysis are quite small (amongst others due to the low frequency of GATA6 amplification). Consequently, large prospective studies are essential for further validation. Finally, mechanistic approaches for further investigation of the biological functions/interactions related to GATA6 amplification in EAC via in-vitro, respectively, in vivo experiments should gain more knowledge about how this molecular alteration might be a target for future treatment concepts.

Author contributions PSP, HL, FG, and AQ conceived and designed the study; PSP, TZ, HA, WS, and FG enrolled the patients and collected the clinical data while HL, AE, and AQ performed the pathological analysis; PSP, and FG carried out the statistical analyses; PSP, HL, TZ,CJB, AMH, RB, FG, and AQ contributed to the interpretation of data. PSP, FG, and AQ drafted the manuscript; All authors were involved in critically revising the manuscript for important intellectual content, and approving of the submitted version. 
Funding Open Access funding enabled and organized by Projekt DEAL. The current study was not funded by any organization.

Data availability The datasets generated and/or analyzed during this current study are available from the corresponding author on reasonable request.

\section{Compliance with ethical standards}

Conflict of interest Patrick Sven Plum is fellow of the Else Kröner Forschungskolleg Cologne "Clonal Evolution in Cancer" (2016-Kolleg-19). All other authors declare no conflict of interest.

Ethics approval This retrospective study was performed according to the criteria of the ethics committee of the University Hospital of Cologne (No. 13-091 and 10-242) and in accordance with the relevant version of the Helsinki Declaration.

Consent to participate/publication All patients declared their participation and written consent was obtained before participation in the study (No. 13-091 and 10-242). The objective of the project was primarily in the field of diagnostics and quality assurance.

Open Access This article is licensed under a Creative Commons Attribution 4.0 International License, which permits use, sharing, adaptation, distribution and reproduction in any medium or format, as long as you give appropriate credit to the original author(s) and the source, provide a link to the Creative Commons licence, and indicate if changes were made. The images or other third party material in this article are included in the article's Creative Commons licence, unless indicated otherwise in a credit line to the material. If material is not included in the article's Creative Commons licence and your intended use is not permitted by statutory regulation or exceeds the permitted use, you will need to obtain permission directly from the copyright holder. To view a copy of this licence, visit http://creativecommons.org/licenses/by/4.0/.

\section{References}

Al-Batran S-E, Hartmann JT, Hofheinz R et al (2008) Biweekly fluorouracil, leucovorin, oxaliplatin, and docetaxel (FLOT) for patients with metastatic adenocarcinoma of the stomach or esophagogastric junction: a phase II trial of the Arbeitsgemeinschaft Internistische Onkologie. Ann Oncol Off J Eur Soc Med Oncol 19:1882-1887. https://doi.org/10.1093/annonc/mdn403

Al-Batran SE, Homann N, Pauligk C et al (2019) Perioperative chemotherapy with fluorouracil plus leucovorin, oxaliplatin, and docetaxel versus fluorouracil or capecitabine plus cisplatin and epirubicin for locally advanced, resectable gastric or gastrooesophageal junction adenocarcinoma (FLOT4): a ra. Lancet 393:1948-1957. https://doi.org/10.1016/S0140-6736(18)32557-1

Arnold M, Laversanne M, Brown LM et al (2017) Predicting the future burden of esophageal cancer by histological subtype: international trends in incidence up to 2030. Am J Gastroenterol 112:12471255. https://doi.org/10.1038/ajg.2017.155

Becker J, May A, Gerges C et al (2015) Supportive evidence for FOXP1, BARX1, and FOXF1 as genetic risk loci for the development of esophageal adenocarcinoma. Cancer Med 4:1700-1704. https://doi.org/10.1002/cam4.500

Cancer genome Atlas research network, analysis working group: asan university, BC cancer agency et al (2017) Integrated genomic characterization of oesophageal carcinoma. Nature 541:169-175. https://doi.org/10.1038/nature20805

Carrasco M, Delgado I, Soria B et al (2012) GATA4 and GATA6 control mouse pancreas organogenesis. J Clin Invest 122:3504-3515. https://doi.org/10.1172/JCI63240

Coleman HG, Xie SH, Lagergren J (2018) The epidemiology of esophageal adenocarcinoma. Gastroenterology 154:390-405. https:// doi.org/10.1053/j.gastro.2017.07.046

den Bakker CM, Smit JK, Bruynzeel AME et al (2017) Non responders to neoadjuvant chemoradiation for esophageal cancer: Why better prediction is necessary. J Thorac Dis 9:S843-S850. https://doi. org/10.21037/jtd.2017.06.123

DeSantis CE, Lin CC, Mariotto AB et al (2014) Cancer treatment and survivorship statistics, 2014. CA Cancer J Clin 64:252-271. https ://doi.org/10.3322/caac.21235

Donohoe CL, Reynolds JV (2017) Neoadjuvant treatment of locally advanced esophageal and junctional cancer: the evidence-base, current key questions and clinical trials. J Thorac Dis 9:S697S704. https://doi.org/10.21037/jtd.2017.03.159

Dulak AM, Stojanov P, Peng S et al (2013) Exome and wholegenome sequencing of esophageal adenocarcinoma identifies recurrent driver events and mutational complexity. Nat Genet 45:478-486. https://doi.org/10.1038/ng.2591

Essakly A, Loeser H, Kraemer M et al (2020) PIK3CA and KRAS amplification in esophageal adenocarcinoma and their impact on the inflammatory tumor microenvironment and prognosis. Transl Oncol 13:157-164. https://doi.org/10.1016/j.trano n.2019.10.013

Frankell AM, Jammula SG, Li X et al (2019) The landscape of selection in 551 esophageal adenocarcinomas defines genomic biomarkers for the clinic. Nat Genet 51:506-516. https://doi. org/10.1038/s41588-018-0331-5

Gebauer F, Krämer M, Bruns C et al (2020) Lymphocyte activation gene-3 (LAG3) mRNA and protein expression on tumour infiltrating lymphocytes (TILs) in oesophageal adenocarcinoma. J Cancer Res Clin Oncol 146:2319-2327. https://doi.org/10.1007/ s00432-020-03295-7

Helbig D, Ihle MA, Pütz K et al (2016) Oncogene and therapeutic target analyses in Atypical fibroxanthomas and pleomorphic dermal sarcomas. Oncotarget 7:21763-21774. https://doi.org/10.18632/ oncotarget.7845

Jimenez P, Saner K, Mayhew B, Rainey WE (2003) GATA-6 is expressed in the human adrenal and regulates transcription of genes required for adrenal androgen biosynthesis. Endocrinology 144:4285-4288. https://doi.org/10.1210/en.2003-0472

Kamnasaran D, Guha A (2005) Expression of GATA6 in the human and mouse central nervous system. Dev Brain Res 160:90-95. https://doi.org/10.1016/j.devbrainres.2005.07.012

Lin BAJ, Lockwood WW et al (2012) Activation of GATA binding protein 6 (GATA6) sustains oncogenic lineage-survival in esophageal adenocarcinoma. Proc Natl Acad Sci USA 109:4251-4256. https ://doi.org/10.1073/pnas.1011989109

Liu H, Du F, Sun L et al (2019) GATA6 suppresses migration and metastasis by regulating the miR-520b/CREB 1 axis in gastric cancer. Cell Death Dis 10:35. https://doi.org/10.1038/s4141 9-018-1270-x

Loeser H, Kraemer M, Gebauer F et al (2019) The expression of the immune checkpoint regulator VISTA correlates with improved overall survival in pT1/2 tumor stages in esophageal adenocarcinoma. Oncoimmunology 8:1-8. https://doi.org/10.1080/21624 02X.2019.1581546

Ma R, Li X, Liu H et al (2019) GATA6-upregulating autophagy promotes TKI resistance in nonsmall cell lung cancer. Cancer Biol Ther 20:1206-1212. https://doi.org/10.1080/15384 047.2019 .1599665 
Mariette C, Markar SR, Dabakuyo-Yonli TS et al (2019) Hybrid minimally invasive esophagectomy for esophageal cancer. $\mathrm{N}$ Engl J Med 380:152-162. https://doi.org/10.1056/nejmoa1805101

Mourikis TP, Benedetti L, Foxall E et al (2019) Patient-specific cancer genes contribute to recurrently perturbed pathways and establish therapeutic vulnerabilities in esophageal adenocarcinoma. Nat Commun 10:3101. https://doi.org/10.1038/s41467-019-10898-3

Nones K, Waddell N, Wayte N et al (2014) Genomic catastrophes frequently arise in esophageal adenocarcinoma and drive tumorigenesis. Nat Commun 5:5224. https://doi.org/10.1038/ncomms6224

Pavlov K, Honing J, Meijer C et al (2015) GATA6 expression in Barrett's oesophagus and oesophageal adenocarcinoma. Dig Liver Dis 47:73-80. https://doi.org/10.1016/j.dld.2014.09.014

Plum PS, Hölscher AH, Pacheco Godoy K et al (2018) Prognosis of patients with superficial T1 esophageal cancer who underwent endoscopic resection before esophagectomy-A propensity scorematched comparison. Surg Endosc 32:3972-3980. https://doi. org/10.1007/s00464-018-6139-7

Plum PS, Gebauer F, Krämer M et al (2019) HER2/neu (ERBB2) expression and gene amplification correlates with better survival in esophageal adenocarcinoma. BMC Cancer 19:38. https://doi. org/10.1186/s 12885-018-5242-4

Rao X, Wan L, Jie Z et al (2019) Upregulated miR-27a-3p indicates a poor prognosis in pancreatic carcinoma patients and promotes the angiogenesis and migration by epigenetic silencing of GATA6 and activating VEGFA/VEGFR2 signaling pathway. Onco Targets Ther 12:11241-11254. https://doi.org/10.2147/OTT.S220621

Ronellenfitsch U, Liodaki E, Trunk MJ et al (2016) Association between tumor response and postoperative morbidity after neoadjuvant chemotherapy for gastroesophageal adenocarcinoma? J Unexplored Med Data 1:6-14. https://doi.org/10.20517 /2572-8180.2016.01

Rustgi AK, El-Serag HB (2014) Esophageal carcinoma. N Engl J Med 371:2499-2509. https://doi.org/10.1056/NEJMra1314530

Schallenberg S, Bork J, Essakly A et al (2020) Loss of the SWI/SNFATPase subunit members SMARCF1 (ARID1A), SMARCA2 (BRM), SMARCA4 (BRG1) and SMARCB1 (INI1) in oesophageal adenocarcinoma. BMC Cancer 20:1-12. https://doi. org/10.1186/s12885-019-6425-3

Schiffmann LM, Loeser H, Jacob AS et al (2020) Dickkopf-2 (DKK2) as context dependent factor in patients with esophageal adenocarcinoma. Cancers (Basel). https://doi.org/10.3390/cancers120 20451

Shapiro J, van Lanschot JJB, Hulshof MCCM et al (2015) Neoadjuvant chemoradiotherapy plus surgery versus surgery alone for oesophageal or junctional cancer (CROSS): long-term results of a randomised controlled trial. Lancet Oncol 16:1090-1098. https ://doi.org/10.1016/S1470-2045(15)00040-6
Shen F, Li J, Cai W et al (2013) GATA6 predicts prognosis and hepatic metastasis of colorectal cancer. Oncol Rep 30:1355-1361. https:// doi.org/10.3892/or.2013.2544

Shen W, Niu N, Lawson B et al (2019) GATA6: a new predictor for prognosis in ovarian cancer. Hum Pathol 86:163-169. https://doi. org/10.1016/j.humpath.2019.01.001

Simon R, Mirlacher M, Sauter G (2005) Tissue microarrays. Methods Mol Med 114:257-268. https://doi.org/10.1385/1-59259 $-923-0: 257$

Suzuki E, Evans T, Lowry J et al (1996) The human GATA-6 gene: Structure, chromosomal location, and regulation of expression by tissue-specific and mitogen-responsive signals. Genomics 38:283-290. https://doi.org/10.1006/geno.1996.0630

Tian F, Li D, Chen J et al (2013) Aberrant expression of GATA binding protein 6 correlates with poor prognosis and promotes metastasis in cholangiocarcinoma. Eur J Cancer 49:1771-1780. https://doi. org/10.1016/j.ejca.2012.12.015

Toxopeus ELA, Lynam-Lennon N, Biermann K et al (2019) Tumor microRNA-126 controls cell viability and associates with poor survival in patients with esophageal adenocarcinoma. Exp Biol Med 244:1210-1219. https://doi.org/10.1177/1535370219868671

Van Baal JWPM, Verbeek RE, Bus P et al (2013) MicroRNA-145 in Barrett's oesophagus: regulating BMP4 signalling via GATA6. Gut 62:664-675. https://doi.org/10.1136/gutjnl-2011-301061

van Hagen P, Hulshof MCCM, van Lanschot JJB et al (2012) Preoperative chemoradiotherapy for esophageal or junctional cancer. N Engl J Med 366:2074-2084. https://doi.org/10.1056/NEJMo a1112088

von Loga K, Woolston A, Punta M et al (2020) Extreme intratumour heterogeneity and driver evolution in mismatch repair deficient gastro-oesophageal cancer. Nat Commun 11:1-14. https://doi. org/10.1038/s41467-019-13915-7

Wagener-Ryczek S, Schoemmel M, Kraemer M et al (2020) Immune profile and immunosurveillance in treatment-naive and neoadjuvantly treated esophageal adenocarcinoma. Cancer Immunol Immunother 69:523-533. https://doi.org/10.1007/s00262-01902475-w

Zhong Y, Wang Z, Fu B et al (2011) GATA6 activates Wnt signaling in pancreatic cancer by negatively regulating the Wnt antagonist Dickkopf-1. PLoS ONE. https://doi.org/10.1371/journ al.pone. 0022129

Publisher's Note Springer Nature remains neutral with regard to jurisdictional claims in published maps and institutional affiliations. 